\title{
United to preserve antimicrobials
}

Wendy Thompson, Clinical Academic GDP at the University of Manchester and

\{my\}dentist, and advisor to the FDI World Dental Federation on tackling antibiotic resistance

The BDJ Upfront section includes editorials, letters, news, book reviews and interviews. Please direct your correspondence to the News Editor,

Kate Quinlan at k.quinlan@nature.com. Press releases or articles may be edited, and should include a colour photograph if possible.

A $\mathrm{n}$ unintended consequence of the COVID-19 lockdowns in the United Kingdom has been a worrying increase in antibiotic prescribing by dentists. ${ }^{1}$ Antibiotic resistance (ABR) poses a significant threat to global health and wealth, due to prolonged illnesses, longer hospital stays and increased mortality. ${ }^{2}$ The World Health Organisation has highlighted the urgency of tackling ABR by its inclusion in the five platforms to global health and wellbeing. ${ }^{2}$

If $\mathrm{ABR}$ continues to increase, it has been estimated that by 2050 , infections resistant to drugs will be the number one cause of death globally, with 10 million people dying every year. Both the United Nations and the World Bank have highlighted the potential annual economic impact of antimicrobial resistance (up to US\$ 3.4 trillion per year). Low income countries are anticipated to experience a larger impact. ${ }^{2}$

Resistance is driven by the overuse of antibiotics in both people and animals (especially for food production), as well as in the environment. Dentists are responsible for about $10 \%$ of antibiotic prescribing for humans globally, three quarters of which is estimated to be unnecessary. Which is why, after years of a downward trend, the large rise in dental antibiotic prescribing, over 25\% in the three months of April to June, driven by restricted access to dental care due to COVID-19, is a huge concern. ${ }^{1}$

Antibiotics are life-saving drugs; when people really need them, the drugs really need to work. Infections that are resistant to antibiotics therefore pose a serious risk to patient safety. Exposing a patient to antibiotics when not necessary (for example 'just in case' or to meet patient demands) increases that patient's risk of the antibiotics failing when they are necessary. For patients with a spreading dental infection, effective antibiotics are vital; sepsis and the spread of infection toward vital structures may

occur rapidly and these conditions can be life threatening. Ensuring access to quick, appropriate, and effective treatment is extremely important for these patients.

Most dental infections are amenable to treatment by a dental procedure to remove the source of the infection without the need for antibiotics. In normal times, antibioticonly treatment plans are rarely appropriate. Dentists are surgeons, skilled and equipped to diagnose and treat acute dental conditions during urgent appointments. The importance of access to face-to-face dental, rather than medical, care for patients with acute dental

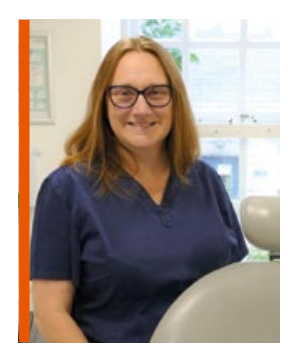

\section{'The dental profession has a clear responsibility to engage, commit and contribute to global, national and local efforts to tackle ABR.'}

conditions is clear. Optimising dental prescribing, at the same time, increases patient safety by reducing the risk of adverse reactions such as anaphylaxis. Reports indicate that dental prescribing contributes to the incidence of Clostridioides difficile (C. difficile) in the general population. ${ }^{2}$ And that is precisely the rub with ABR: infections which are resistant to antibiotics are not just a problem for the individuals who take the antibiotics - their spread is a problem for society as a whole.

We are staring down a long-term pandemic and urgent collective action is needed to slow it down. Moving forward, the dental profession has a clear responsibility to engage, commit and contribute to global, national and local efforts to tackle ABR. In 2019, the FDI World Dental Federation (FDI) highlighted the overwhelming case for improved antibiotic stewardship, with the use of antibiotics restricted to only when strictly necessary. The crucial role of national bodies, local teams and individual prescribers was identified for tackling the global problem of ABR.

The FDI's White Paper, The essential role of the dental team in reducing antibiotic resistance, released this week, is an important step forward in acknowledging that dentists around the world have to be aware of $A B R$, recognised for their role in preventing infections and empowered to optimise their antibiotic prescribing. This is how the profession can play its part in preserving effective antimicrobials.

We live in especially challenging times. Patients waiting for access to the right care often receive more antibiotics than they 